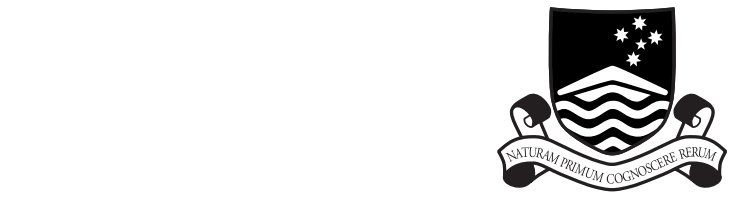

THE AUSTRALIAN NATIONAL UNIVERSITY

TR-CS-97-09

\title{
Constant Time Algorithms for Computing the Contour of Maximal Elements on the Reconfigurable Mesh
}

\author{
M. Manzur Murshed and Richard P. Brent \\ May 1997
}

Joint Computer Science Technical Report Series

Department of Computer Science

Faculty of Engineering and Information Technology

Computer Sciences Laboratory

Research School of Information Sciences and Engineering 
This technical report series is published jointly by the Department of Computer Science, Faculty of Engineering and Information Technology, and the Computer Sciences Laboratory, Research School of Information Sciences and Engineering, The Australian National University.

Please direct correspondence regarding this series to:

Technical Reports

Department of Computer Science

Faculty of Engineering and Information Technology

The Australian National University

Canberra ACT 0200

Australia

or send email to:

Technical.Reports@cs.anu.edu.au

A list of technical reports, including some abstracts and copies of some full reports may be found at:

http://cs.anu.edu.au/techreports/

\section{Recent reports in this series:}

TR-CS-97-08 Xun Qu, Jeffrey Xu Yu, and Richard P. Brent. A mobile TCP socket. April 1997.

TR-CS-97-07 Richard P. Brent. A fast vectorised implementation of Wallace's normal random number generator. April 1997.

TR-CS-97-06 M. Manzur Murshed and Richard P. Brent. RMSIM: a serial simulator for reconfigurable mesh parallel computers. April 1997.

TR-CS-97-05 Beat Fischer. Collocation and filtering - a data smoothing method in surveying engineering and geodesy. March 1997.

TR-CS-97-04 Stephen Fenwick and Chris Johnson. HeROD flavoured oct-trees: Scientific computation with a multicomputer persistent object store. February 1997.

TR-CS-97-03 Brendan D. McKay. Knight's tours of an $8 \times 8$ chessboard. February 1997. 


\title{
Constant Time Algorithms for Computing the Contour of Maximal Elements on the Reconfigurable Mesh
}

\author{
M. Manzur Murshed* \\ Richard P. Brent \\ Computer Sciences Lab, Research School of Information Sciences 83 Engg. \\ Australian National University, Canberra ACT 0200, Australia \\ e-mail:murshed@cslab.anu.edu.au \\ May 12, 1997
}

\section{Abstract}

There has recently been an interest in the introduction of reconfigurable buses to existing parallel architectures. Among them Reconfigurable Mesh (RM) draws much attention because of its simplicity. This paper presents two $O(1)$ time algorithms to compute the contour of the maximal elements of $N$ planar points on the RM. The first algorithm employs an RM of size $N \times N$ while the second one uses a 3 -D RM of size $\sqrt{N} \times \sqrt{N} \times \sqrt{N}$.

\section{Introduction}

It is well-known that interprocessor communications and simultaneous memory accesses often act as bottlenecks in present-day parallel machines. Bus systems have been intro-

\footnotetext{
${ }^{*}$ Corresponding author.
}

duced recently to a number of parallel machines to address this problem. Examples include the Bus Automaton [15], the Reconfigurable Mesh (RM) [11], the content addressable array processor [16], and the Polymorphic torus [9]. A bus system is called reconfigurable if it can be dynamically changed according to either global or local information.

Jang et al. [17] have recently studied a number of constant time computational geometry algorithms on the reconfigurable mesh. In this paper we explore one further problem from a similar point of view. The problem, considered from computational geometry, is to compute the contour of the maximal elements of a given set of planar points (see Section 2.2). Solution to this problem is important in solving the Largest Empty Rectangle Problem $[2,5,14]$ where a rectangle and a number of planar points in it are given and the problem is to compute the largest rectan- 
gle containing no points.

It is well known that the time complexity for computing the contour of the maximal elements of $N$ planar points is $\Theta(N \log N)$ using a sequential computer [7]. Dehne [4] gives an efficient algorithm for solving this problem on a mesh of size $\sqrt{N} \times \sqrt{N}$ in $O(\sqrt{N})$ time. Whenever necessary $N$ is assumed, throughout the paper, to be a perfect square without any loss of generality.

In this paper we present two $O(1)$ time algorithms to compute the contour of the maximal elements of $N$ planar points on the reconfigurable mesh. The first algorithm employs an RM of size $N \times N$ while the second one uses a 3-D RM of size $\sqrt{N} \times \sqrt{N} \times \sqrt{N}$. To our knowledge this problem on the reconfigurable mesh is examined here for the first time.

This paper is organized as follows. In the next section we present the basic issues of RM as well as the definition of the problem. Constant time algorithms are developed in Section 3. Section 4 concludes the paper.

\section{Preliminaries}

For the sake of completeness, we briefly define the reconfigurable mesh and definitions of the problem of computing the contour of the maximal elements of a given set of planar points.

\subsection{Reconfigurable Mesh}

The reconfigurable mesh is primarily a twodimensional mesh of processors connected by

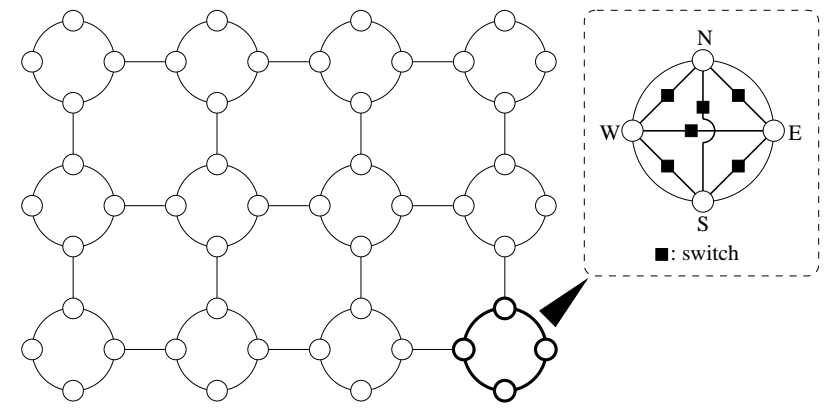

Figure 1: A reconfigurable mesh of size $3 \times 4$

reconfigurable buses. In this parallel architecture, a processor element is placed at the grid points as in the usual mesh connected computers. Processors of the RM of size $X \times Y$ are denoted by $P E_{i, j}, 0 \leq i<X-1$, $0 \leq j<Y-1$. Each processor is connected to at most four neighbouring processors through fixed bus segments connected to four I/O ports $\mathcal{E} \& \mathcal{W}$ along dimension $x$ and $\mathcal{N} \& \mathcal{S}$ along dimension $y$. These fixed bus segments are building blocks of larger bus components which are formed through switching, decided entirely on local data, of the internal connectors (see Figure 1) between the I/O ports of each processor. The fifteen possible interconnections of I/O ports through switching are shown in Figure 2. Like all bus systems, the behaviour of RM relies on the assumption that the transmission time of a message along a bus is independent of the length of the bus [1].

A reconfigurable mesh operates in the single instruction multiple data (SIMD) mode. Besides the reconfigurable switches, each processor has a computing unit with a fixed number of local registers. A single time step of 


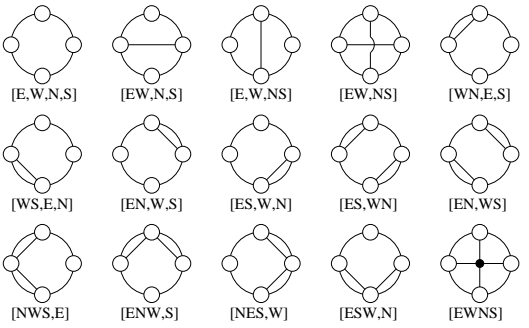

Figure 2: Possible internal connections between the four I/O ports of a processor

an RM is composed of the following four substeps:

BUS substep. Every processor switches the internal connectors between I/O ports by local decision.

WRITE substep. Along each bus, one or more processors on the bus transmit a message of length bounded by the bandwidth of the fixed bus segments as well as the switches. These processors are called the speakers. It is assumed that a collision between several speakers will be detected by all the processors connected to the bus and the transmitted message will be discarded.

READ substep. Some or all the processors connected to a bus read the message transmitted by a single speaker. These processors are called the readers.

COMPUTE substep. A constant-time local computation is done by each processor.

Reconfigurable meshes of higher dimension can be constructed in a similar way. Processors of a 3 -D RM of size $X \times Y \times Z$ are denoted by $P E_{i, j, k}, 0 \leq i<X-1,0 \leq j<Y-1$, $0 \leq k<Z-1$. Each processor of a $3-\mathrm{D}$ RM has two additional ports $\mathcal{U}$ and $\mathcal{D}$ along dimension $z$.

Many basic operations can be performed in constant time on RM. Below we briefly outline the results used in our algorithms in Section 3 .

Given a binary sequence, $b_{j}, 0 \leq j<N$, the prefix-and computation is to compute, $\forall i$ : $0 \leq i<N, b_{0} \wedge b_{1} \wedge \cdots \wedge b_{i}$. Similarly the prefix-or computation computes $b_{0} \vee b_{1} \vee \cdots \vee$ $b_{i}, \forall i: 0 \leq i<N$. Adapting the technique of bus splitting [12] it is easy to show:

Lemma 1 Given a binary sequence of length $N$ in the only row of an $R M$ of size $1 \times N$, both the prefix-and and the prefix-or of the elements in the sequence can be computed in $O(1)$ time.

Given $N$ numbers of $\log N$ bits each, the problem of sorting these numbers on an RM of size $N \times N$ has been considered in $[1,6,8,13]$. Several authors [3, 10, 13] consider an RM of size $\sqrt{N} \times \sqrt{N} \times \sqrt{N}$ to sort $N$ numbers. It is easy to show that these algorithms can be modified to sort $N$ constant length records within a constant time reduction. Here the following lemmas are stated without proof.

Lemma 2 Given $N$ constant length records in a row, these records can be sorted in $O(1)$ time using an $R M$ of size $N \times N$.

Lemma 3 Given $N$ constant length records in a plane, these records can be sorted in $O(1)$ time using an $R M$ of size $\sqrt{N} \times \sqrt{N} \times \sqrt{N}$. 


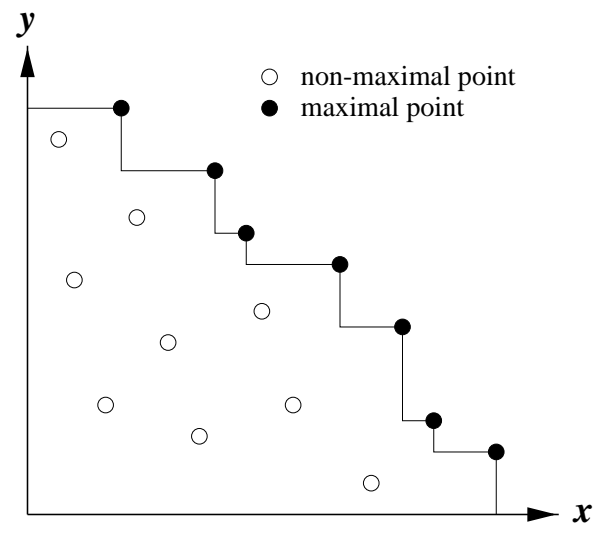

Figure 3: m-contour of a set of planar points

\subsection{Problem Definition}

Let the planar point at coordinate $(i, j)$ be defined as $P(i, j)$. Again, let for any point $p$, $x(p)$ denote the $x$-coordinate and $y(p)$ denote the $y$-coordinate of $p$, e.g., $x(P(i, j))=i$ and $y(P(i, j))=j$.

Definition 1 A point $p$ dominates a point $q$ (denoted by $q \prec p$ ) if $x(q) \leq x(p)$ and $y(q) \leq$ $y(p)$. (The relation “ $\prec$ " is naturally called dominance.)

Let $S$ be a set of $N$ planar points. To simplify the exposition of our algorithms, the points in $S$ are assumed to be distinct.

Definition $2 A$ point $p \in S$ is maximal if there is no other point $q \in S$ with $p \prec q$.

We are interested in the contour spanned by the maximal elements of $S$, called the $m$ contour of $S$ which can be obtained by simply sorting the maximal elements in ascending order of their $x$-coordinates (Figure 3 ). Let the m-contour of a set $S$ be denoted as $m(S)$.
Two interesting observations on mcontour, connected to our algorithms, are given below:

Lemma 4 Every m-contour is sorted in descending order of the $y$-coordinates.

Proof. Suppose the contrary. Then there exists at least one pair of maximal elements $p$ and $q$ such that $y(p)<y(q)$ while $x(p) \leq$ $x(q)$, which contradicts with the assumption that point $p$ is maximal.

Let for any set $S$ of some planar points functions $\min _{x}(S)$ and $\max _{x}(S)$ denote the minimum and maximum $x$-coordinatesin the set respectively. Let two more functions $\min _{y}(S)$ and $\max _{y}(S)$ be defined similarly w.r.t. $y$-coordinate.

Lemma 5 Given $K$ sets $s_{0}, s_{1}, \ldots s_{K-1}$ of planar points such that $\forall k: 0 \leq k<K-1$, $\max _{x}\left(s_{k}\right) \leq \min _{x}\left(s_{k+1}\right)$, then $\forall i: 0 \leq i<$ $K-1, \forall p \in m\left(s_{i}\right) \wedge y(p)>\max _{y}\left(m\left(s_{j}\right)\right)$, $\forall j>i$, if and only if, $p \in m\left(\bigcup_{k=0}^{K-1} s_{k}\right)$.

Proof. The necessity part can be proved by arranging a contradiction of Lemma 4 . To prove the sufficiency part we take a point $p \in$ $m\left(s_{i}\right), \exists i: 0 \leq i<K-1 \wedge p \notin m\left(\bigcup_{k=0}^{K-1} s_{k}\right)$. Then by the definition of maximality we get $\exists q \in \bigcup_{k=i+1}^{K-1} s_{k}$ such that $p \prec q$, i.e., $y(p) \leq$ $y(q)$.

\section{Constant Time Algo- rithms for Computing m-contour}

We develop two constant time algorithms for computing the m-contour of a set of $N$ planar 
points. The first algorithm MAXIMAL1 uses a 2-D RM of size $N \times N$ while the second algorithm MAXIMAL2 requires a 3-D RM of size $\sqrt{N} \times \sqrt{N} \times \sqrt{N}$.

\subsection{First Algorithm}

The algorithm MAXIMAL1 is very straightforward. $N$ planar points are given in the $N$ processors at row 0 . These points, after sorting, are distributed over the RM through column and row broadcast in such a way that each column $i, 0 \leq i,<N$, of the RM computes the dominance of all other points over the $i$ th point. Then each column computes the logical or of the previous dominance decision to assert whether the point represented by that column is a maximal point or not. As all the points are already sorted, the mcontour is obtained simply by following this sorted sequence. The detailed description of the algorithm is given below. Here and below we assume the following while presenting our algorithms for RM:

- In every step there may be at most four substeps labelled as "b:", "w:", "r:", and "c:" for the BUS, WRITE, READ, and COMPUTE substeps respectively.

- Any step without any labelled substep means the use of another algorithm, internal or external to this paper.

- In any step if the BUS substep is missing for a particular processor it is assumed that the local port interconnections of that processor remain unchanged.

\section{Algorithm: MAXIMAL1}

Precondition: registers $r_{0}$ and $r_{1}$ hold $x$ and $y$-coordinates respectively.

Postcondition: register $r_{2}$ holds the decision of maximality.

1. Sort the given $N$ points at row 0 in ascending order of register $r_{0}$, i.e., in ascending order of the $\mathrm{x}$-coordinates. The sorted list resides in the processors of row 0 in the ascending row-major order.

2. b: Every processor connects port $\mathcal{N}$ with $\mathcal{S}$;

w: Every processor at row 0 writes register $r_{0}$ to port $\mathcal{N}$;

r: Every processor reads port $\mathcal{N}$ into register $r_{0}$;

3. w: Every processor at row 0 writes register $r_{1}$ to port $\mathcal{N}$;

r: Every processor reads port $\mathcal{N}$ into register $r_{1}$;

4. b: Every processor connects port $\mathcal{E}$ with $\mathcal{W}$;

w: Every processor $P E_{i, i}, 0 \leq i<N$, writes register $r_{0}$ to port $\mathcal{E}$;

r: Every processor reads port $\mathcal{E}$ into register $r_{2}$;

5. w: Every processor $P E_{i, i}, 0 \leq i<N$, writes register $r_{1}$ to port $\mathcal{E}$;

r: Every processor reads port $\mathcal{E}$ into register $r_{3}$; 
6. b: Every processor does the following: if $P\left(r_{0}, r_{1}\right) \prec P\left(r_{2}, r_{3}\right)$ then disconnect all the ports;

$$
\text { else }
$$$$
\text { connect port } \mathcal{N} \text { with } \mathcal{S} \text {; }
$$

w: Every processor at row $N-1$ writes an arbitrary constant \# to port $\mathcal{N}$;

r: Every processor at row 0 reads port $\mathcal{S}$ into register $r_{2} ;$

c: Every processor at row 0 does the following:

$$
\begin{aligned}
& \text { if } r_{2}=\# \text { then } \\
& \text { set register } r_{2}=1 \text {; } \\
& \text { else }
\end{aligned}
$$$$
\text { set register } r_{2}=0 \text {; }
$$

Theorem 1 Given $N$ planar points in a row, the $m$-contour of these points can be obtained in $O(1)$ time using an $R M$ of size $N \times N$.

Proof. Step 1 of algorithm MAXIMAL1 can be computed in constant time using Lemma 2. Steps 2-5 require $O(1)$ time. Step 6 is an elaboration of computing prefixor partially and requires constant time by Lemma 1.

\subsection{Second Algorithm}

In algorithm MAXIMAL2 we use the wellknown divide-and-conquer approach to compute the m-contour. $N$ points are given in the $(X Y-\text { plane })_{z=0}$. These points are sorted in order of $x$-coordinate to divide them into $\sqrt{N}$ disjoint sets of length $\sqrt{N}$ each. This division complies with the first condition in Lemma 5. Now the m-contour of these smaller sets are computed using all the 2-D submeshes lying parallel to $Y Z$-plane. Now merging of the solutions of these smaller problems is done by carefully utilizing Lemma 5 . Lemma 4 helps in getting the $\max _{y}$ of each smaller m-contour in constant time (Step 3). The $i$ th $\max _{y}$ is then distributed over the (XYplane $)_{z=i}$ (Step 4-6). Every point in each smaller m-contour then computes its overall maximality using the processors along the $z$ axis (Step 7-8). The detailed description of the algorithm is given below.

\section{Algorithm: MAXIMAL2}

Precondition: registers $r_{0}$ and $r_{1}$ hold $x$ and $y$-coordinates respectively.

Postcondition: register $r_{2}$ holds the decision of maximality.

1. Sort the given $N$ points in the (XYplane $)_{z=0}$ in ascending order of register $r_{0}$, i.e., in ascending order of the $\mathrm{x}$ coordinates. The sorted list resides in the processors of the $(X Y-\text { plane })_{z=0}$ in ascending column-major order.

2. For every column $i, 0 \leq i<\sqrt{N}$, of $(X Y-\text { plane })_{z=0}$ compute the m-contour of the $\sqrt{N}$ points residing in processors $P E_{i, j, 0}, 0 \leq j<\sqrt{N}$, using the algorithm MAXIMAL1 on the 2-D submesh lying in the $(Y Z-\text { plane })_{x=i}$.

3. b: Every processor in the $(X Y-\text { plane })_{z=0}$ does the following:

$$
\text { if } r_{2}=0 \text { then }
$$


connect port $\mathcal{N}$ with $\mathcal{S}$;

else

disconnect all the ports;

w: Every processor in the

$(X Y-\text { plane })_{z=0}$ does the

following:

if $r_{2}=1$ then

write register $r_{1}$ to port $\mathcal{S}$;

r: Every processor $P E_{i, 0,0}, 0 \leq i<$ $\sqrt{N}$, reads port $\mathcal{S}$ into register $r_{3}$;

4. b: Every processor $P E_{i, 0, k}, 0 \leq i, k<$ $\sqrt{N}$, connects port $\mathcal{U}$ with $\mathcal{D}$;

w: Every processor $P E_{i, 0,0}, 0 \leq i<$ $\sqrt{N}$, writes register $r_{3}$ to port $\mathcal{U}$;

r: Every processor $P E_{i, 0, i}, 0 \leq i<$ $\sqrt{N}$, reads port $\mathcal{U}$ into register $r_{3}$;

5. b: Every processor in the $(X Z-$ plane $)_{y=0}$ connects port $\mathcal{E}$ with $\mathcal{W}$;

w: Every processor $P E_{i, 0, i}, 0 \leq i<$ $\sqrt{N}$, writes register $r_{3}$ to port $\mathcal{E}$;

$\mathrm{r}$ : Every processor in the $(X Z-$ plane $)_{y=0}$ reads port $\mathcal{E}$ into register $r_{3}$

6. b: Every processor connects port $\mathcal{N}$ with $\mathcal{S}$;

w: Every processor in the $(X Z-$ plane) ${ }_{y=0}$ writes register $r_{3}$ to port $\mathcal{N}$

r: Every processor reads port $\mathcal{N}$ into register $r_{3}$;

7. b: Every processor connects port $\mathcal{U}$ with $\mathcal{D}$; w: Every processor in the $(X Y-$ plane $)_{z=0}$ writes register $r_{1}$ to port $\mathcal{U}$;

r: Every processor reads port $\mathcal{U}$ into register $r_{4}$;

8. b: Every processor $P E_{i, j, k}$, $0 \leq i, j, k<\sqrt{N}$, does the following:

if $k>i$ then

if $r_{4}>r_{3}$ then connect port $\mathcal{U}$ with $\mathcal{D}$;

else

disconnect all the ports;

else

connect port $\mathcal{U}$ with $\mathcal{D}$;

$\mathrm{w}$ : Every processor in the $(X Y-$ plane $)_{z=\sqrt{N}-1}$ writes an arbitrary constant \# to port $\mathcal{U}$;

$\mathrm{r}$ : Every processor in the $(X Y-$ plane $)_{z=0}$ reads port $\mathcal{D}$ into register $r_{4}$;

c: Every processor in the $(X Y-\text { plane })_{z=0}$ does the following:

$$
\text { if } r_{2}=1 \text { and } r_{4} \neq \# \text { then }
$$
set register $r_{2}=0$;

Theorem 2 Given $N$ planar points in a plane, the m-contour of these points can be obtained in $O(1)$ time using an $R M$ of size $\sqrt{N} \times \sqrt{N} \times \sqrt{N}$.

Proof. Step 1 of algorithm MAXIMAL2 can be computed in constant time using Lemma 3. By Theorem 1 step 2 can also be done in constant time. It is obvious that the rest of the steps require $O(1)$ time. 


\section{Conclusion}

In this paper we have described two $O(1)$ time algorithms for computing the contour of the maximal elements of a given set of planar points. Hopefully these algorithms will have an application to solving the largest empty rectangle problem in constant time.

\section{References}

[1] Y. Ben-Asher, D. Peleg, R. Ramaswami, and A. Schuster. The power of reconfiguration. Journal of Parallel and Distributed Computing, 13:139-153, 1991.

[2] B. Chazelle, R. L. Drysdale, and D. T. Lee. Computing the largest empty rectangle. SIAM J. Comput., 15:300-315, 1986.

[3] Yen-Cheng Chen and Wen-Tsuen Chen. Constant time sorting on reconfigurable meshes. IEEE Transactions on Computers, 43:749-751, 1994.

[4] Frank Dehne. $O\left(n^{1 / 2}\right)$ algorithms for the maximal elements and ECDF searching problem on a mesh-connected parallel computer. Information Processing Letters, 22:303-306, 1986.

[5] Frank Dehne. Computing the largest empty rectangle on one- and twodimensional processor arrays. Journal of Parallel and Distributed Computing, 9:63-68, 1990.
[6] Ju-Wook Jang and Viktor K. Prasanna. An optimal sorting algorithm on reconfigurable mesh. Journal of Parallel and Distributed Computing, 25:31-41, 1995.

[7] H. T. Kung, F. Luccio, and F. P. Preparata. On finding the maxima of a set of vectors. J. ACM, 22:469-476, 1975 .

[8] R Lin, Stephan Olariu, J. Schwing, and J. Zhang. A VLSI-optimal constant time sorting on reconfigurable mesh. In Ninth European Workshop Parallel Computing, pages 1-16, Spain, 1992.

[9] Massimo Maresca. Polymorphic processor arrays. IEEE Transactions on Parallel and Distributed Systems, 4:490-506, 1993.

[10] Mark S. Merry and Johnnie Baker. A constant time sorting algorithm for a three dimensional reconfigurable mesh and reconfigurable network. Parallel Processing Letters, 5:401-412, 1995.

[11] Russ Miller, V. K. Prasanna Kumar, Dionisios I. Reisis, and Quentin F. Stout. Data movement operations and applications on reconfigurable VLSI arrays. In Proc. International Conference on Parallel Processing, pages 205-208, 1988.

[12] Russ Miller, V. K. Prasanna-Kumar, Dionisios I. Reisis, and Quentin F. Stout. Parallel computations on reconfigurable meshes. IEEE Transactions on Computers, 42:678-692, 1993. 
[13] Madhusudan Nigam and Sartaj Sahni. Sorting $n$ numbers on $n \times n$ reconfigurable meshes with buses. Journal of Parallel and Distributed Computing, 23:37-48, 1994.

[14] M. Orlowski. A new algorithm for the largest empty rectangle problem. Algorithmica, 5:65-73, 1990.

[15] J. Rothstein. Bus automata, brains, and mental models. IEEE Trans. Syst. Man Cybern, 18:522-531, 1988.

[16] C. C. Weems et al. The image understanding architecture. Internat. J. of Comput. Vision, 2:251-282, 1989.

[17] Ju wook Jang, Madhusudan Nigam, Viktor K. Prasanna, and Sartaj Sahni. Constant time algorithms for computational geometry on the reconfigurable mesh. IEEE Transactions on Parallel and Distributed Systems, 8:1-12, 1997. 